\title{
How closely do baryons follow dark matter on large scales?
}

\author{
Raul E. Angulo ${ }^{1,2} *$, Oliver Hahn ${ }^{3,1} \dagger$, Tom Abel ${ }^{1} \ddagger$. \\ ${ }^{1}$ Kavli Institute for Particle Astrophysics and Cosmology, \\ Stanford University, SLAC National Accelerator Laboratory, Menlo Park, CA 94025, USA \\ 2 Max-Planck-Institut für Astrophysik, Karl-Schwarzschild-Strasse 1, 85740 Garching bei München, Germany. \\ 3 Institute for Astronomy, ETH Zurich, CH-8093 Zürich, Switzerland.
}

12 June 2018

\begin{abstract}
We investigate the large-scale clustering and gravitational interaction of baryons and dark matter (DM) over cosmic time using a set of collisionless N-body simulations. Both components, baryons and DM, are evolved from distinct primordial density and velocity power spectra as predicted by early-universe physics. We first demonstrate that such two-component simulations require an unconventional match between force and mass resolution (i.e. force softening on at least the mean particle separation scale). Otherwise, the growth on any scale is not correctly recovered because of a spurious coupling between the two species at the smallest scales. With these simulations, we then demonstrate how the primordial differences in the clustering of baryons and DM are progressively diminished over time. In particular, we explicitly show how the BAO signature is damped in the spatial distribution of baryons and imprinted in that of DM. This is a rapid process, yet it is still not fully completed at low redshifts. On large scales, the overall shape of the correlation function of baryons and DM differs by $\sim 2 \%$ at $z=9$ and by $0.2 \%$ at $z=0$. The differences in the amplitude of the BAO peak are approximately a factor of 5 larger: $10 \%$ at $z=9$ and $1 \%$ at $z=0$. These discrepancies are, however, smaller than effects expected to be introduced by galaxy formation physics in both the shape of the power spectrum and in the BAO peak, and are thus unlikely to be detected given the precision of the next generation of galaxy surveys. Hence, our results validate the standard practice of modelling the observed galaxy distribution using predictions for the total mass clustering in the Universe.
\end{abstract}

Key words: cosmology:theory - large-scale structure of Universe.

\section{INTRODUCTION}

'In the standard paradigm of cosmological structure formation, primordial density perturbations are a result of quantum fluctuations amplified by cosmic inflation. At these very early times, baryons and dark matter (DM) density fields have the 'same phases and amplitudes - each of them has a fluctuation spectrum following a power law with an index close to unity. However, subsequent interaction with the radiation field breaks the initial similarity, creating a scale-dependent growth that is different for baryons and for DM.

On scales smaller than the horizon and prior to recombination, baryons couple to photons through Compton scattering. Radiation pressure opposes gravity and inhibits the growth of density perturbations. The balance is not perfect however, thus generating oscillations on sub-horizon scales in the density, temperature and size of perturbations in the

\footnotetext{
* reangulo@stanford.edu

† hahn@phys.ethz.ch

† tabel@stanford.edu
}

baryon-photon fluid. On even smaller scales, free-streaming and an imperfect coupling between baryons and photons progressively damps the amplitude of these oscillations. In contrast to baryons, DM particles do not directly interact with photons, and are thus mainly affected by gravity. DM density fluctuations can grow freely, and are only halted by the Meszaros effect on scales smaller than the horizon at the matter-radiation equality. The physics describing these interactions is understood at high precision and is able to describe at very high accuracy the patterns of temperature fluctuations observed in the cosmic microwave background radiation (see e.g. $\mathrm{Hu} \&$ Dodelson 2002, for a review).

After recombination, baryons decouple from the photons leading to a drop in sound speed by $\sim 5$ orders of magnitude and an associated drop in the Jeans mass of $\sim 14$ orders of magnitude. From now on, the evolution of perturbations in baryons and DM is dominated by the same physics and the growth is almost entirely determined by gravity until much later times (when hydrodynamical interactions become important). The initial conditions for the two components after 
recombination are, however, very different. The coupling between baryons and photons has prevented the baryons from falling into the density fluctuations present in the DM fluid. The power spectrum of density fluctuations in baryons and DM are thus genuinely distinct: for instance, baryonic acoustic oscillations (BAO) dominate the baryon density power spectrum but they are almost non-existent in the DM distribution.

At later times, the gravitational coupling between DM and baryons will reduce such differences. The DM distribution gradually obtains a BAO signal, while the amplitude of BAOs in the baryons clustering is reduced. This process is commonly assumed to be finished at low redshift, i.e. baryons and DM are assumed to have identical spatial distributions (equal to that of the total mass field) on large scales. A natural corollary of this is that the BAO should be detectable in the galaxy distribution. This has indeed been achieved observationally with increasing accuracy (e.g. Cole et al. 2005; Eisenstein et al. 2005; Blake et al. 2011; Beutler et al. 2011) and further measurements have been proposed using virtually any known tracer of the matter density field in the Universe: including the galaxy distribution (e.g. Coorav 2002), galaxy clusters (e.g Angulo et al. 2005), the $L y-\alpha$ forest (e.g. White et al. 2010; Kitaura et al. 2012) or $21 \mathrm{~cm}$ emission background from galaxies at low redshifts (Chang et al. 2008), from the epoch of re-ionisation at $z \sim 10$ (Mao \& Wu 2008; Rhook et al. 2009), and even using Supernovae (Zhan et al. 2008).

However, the details of the process in which the DM (and thus the total matter) distribution acquires the BAO signature still remain relatively unexplored. At high redshift or large scales, the interaction between baryons and DM particles can be followed accurately by perturbation theory (Somogyi \& Smith 2010; Bernardeau et al. 2012). However, to explore low redshifts and small scales in the mass field, Nbody simulations are essential, since they provide the most accurate and faithful predictions in the nonlinear regime (see Kuhlen et al. 2012, for a recent review). Unfortunately, to our knowledge, no N-body simulation has been performed to address this topic. This is an important issue, since a precise understanding of the BAO signal in the $z \lesssim 10$ Universe is required to interpret accurately the high precision measurements that will be carried out over the next decade.

In this paper, we directly follow the gravitational interaction of DM particles and baryons, from $z=130$ up to the present day. For this, we perform N-body simulations of two interacting fluids with different primordial density and velocity fluctuations: one representing the DM field, and another representing the baryons. We adopt a canonical cosmological model $\Omega_{m}=0.276, \Omega_{\Lambda}=0.724, \Omega_{b}=0.045, h=0.703$, $\sigma_{8}=0.811, n_{s}=0.961$ (Komatsu et al. 2011). We provide details of these simulations and the numerical set-up in $\S 2$. With these simulations in hand, in $\S 3$, we explore the evolution of the large-scale clustering of baryons and DM, with particular emphasis on the evolution of the BAO peak. We discuss our results and conclude in $\S 4$.

\section{N-BODY SIMULATIONS}

\subsection{Initial conditions}

We generate the initial position and velocity for our simulation particles at $z=130$ using the Music code (Hahn \& Abel 2011), and adopting a set of cosmological parameters con- sistent with the published measurements of the WMAP7 data release (Komatsu et al. 2011). Explicitly: $\Omega_{m}=0.276$, $\Omega_{\Lambda}=0.724, \Omega_{b}=0.045, h=0.703, \sigma_{8}=0.811$ and spectral index $n_{s}=0.961$.

We compute the primordial power spectra for baryons and DM using a linear Boltzmann solver code similar to that of Ma \& Bertschinger (1995) where residual baryon-radiation interaction effects become small. The velocity field is irrotational and thus fully described by the velocity divergence, so that the initial conditions are fully specified by the power spectra of the overdensity $\delta$ and the velocity divergence $\theta: P_{\delta_{C}}$, $P_{\theta_{C}}, P_{\delta_{B}}, P_{\theta_{B}}$, where the subscript 'C' stands for CDM and 'B' for baryons.

These power spectra are used together with the Zel'dovich approximation (ZA, i.e. first order Lagrangian Perturbation Theory Zel'Dovich 1970) in Music to generate the initial conditions of our simulations. The gravitational potential, whose gradient appears in both the particles displacement and velocity, is herein replaced by four potentials generated by the respective density power spectra and velocity divergence spectra. Hence, the initial positions and velocities of, for example, baryons is give by:

$$
\mathbf{x}_{B}=\mathbf{q}+\nabla \Phi_{B}, \quad \mathbf{v}_{B}=\nabla \Psi_{B},
$$

where $\mathbf{q}$ is the Lagrangian coordinate and

$$
\begin{aligned}
\Phi_{B}(\mathbf{k}) & \propto \mathcal{G}(\mathbf{k}) k^{-2} \sqrt{P_{\delta_{B}}(k, t)}, \quad \text { and } \\
\Psi_{B}(\mathbf{k}) & \propto \mathcal{G}(\mathbf{k}) k^{-2} \sqrt{P_{\theta_{B}}(k, t)}
\end{aligned}
$$

are the respective potentials and $\mathcal{G}$ is a real-valued Gaussian random field of zero mean and unit variance. In this formulation the streaming velocity between baryons and DM is included self-consistently, but not its non-linear impact onto the spectra until $z=130$, which however is negligibly small on the large scales we consider.

Due to the simplicity and unambiguity in the formulation of two-fluids initial conditions within the ZA, we refrain here from employing the more accurate 2LPT (2nd order Lagrangian Perturbation Theory) formalism (Scoccimarro 1998). In addition, we expect the artefacts and inaccuracies introduced by the ZA to have little impact in our results. This in part because the high starting redshift of our runs, which makes transient features introduced by the ZA have longer time to decay in amplitude. And also because we will be mostly concerned in fractional differences in the measured clustering, thus small artefacts in the clustering partially cancel out.

We also note that at a redshift of 130 there remains a small contribution of the radiation energy density to the Friedmann equation. We ignore this in our simulations and linear theory calculations, setting $\Omega_{r}=0$ at $z \leqslant 130$, but not before.

The baryonic particles are placed on a staggered initial mesh with respect to the DM particles. The required phase shift of the noise field is computed in Fourier space. Staggering is necessary to minimise a spuriously tight coupling between the two particle types (cf. Yoshida et al. 2003, but also our discussion in Section 2.3). We also note that the initial conditions for all our simulations use the same Gaussian white noise field, and thus the resulting cosmic density fields have identical phases. This allows a more accurate comparison between different runs because the effect of cosmic variance is largely reduced. 


\subsection{Specifics of the N-body simulations}

We use two sets of cosmological N-body simulations to study the gravitational coupling of baryons and DM. In the first set, all matter is represented by a single fluid sampling the total matter power spectrum. The second set contains simulations with two distinct fluids, one representing baryons and the other DM, both of which have different primordial density and velocity fluctuations, as predicted by early universe physics. Each set consists of two simulations with different box sizes: i) $L=1 h^{-1} \mathrm{Gpc}$, sufficient to measure reliably the BAO signal; and ii) $L=250 h^{-1} \mathrm{Mpc}$, with which we focus on smaller scales and on the coupling between baryons and DM in the nonlinear regime. In all cases, the DM and baryonic field was each represented with $1024^{3}$ particles, which for the $1 h^{-1} \mathrm{Gpc}$ box implies a DM particle mass of $5.97 \times 10^{10} h^{-1} M_{\odot}$ and a baryon particle mass of $1.16 \times 10^{10} h^{-1} M_{\odot}$. For the smaller simulation box these values are 64 times smaller: $9.3 \times 10^{8} h^{-1} M_{\odot}$ and $1.81 \times 10^{8} h^{-1} M_{\odot}$, respectively. Additionally, for the tests presented in $\S 2.3$ we will employ another set of simulations of mass resolution identical to the $1 h^{-1} \mathrm{Gpc}$ run, but on a $L=500 h^{-1} \mathrm{Mpc}$ box.

We follow the non-linear evolution of the two fluids using a memory-efficient version of the P-Gadget3 Tree-PM code Springel et al. 2005) described in Angulo et al. (2012). We compute only gravitational interactions, neglecting all hydrodynamical ones, thus baryons behave as a collisionless fluid. We expect this to be a good first approximation on the large scales and for the processes we explore here (this approximation is also adopted in perturbation theory). While there are works studying how cooling, star formation and feedback affect the matter power spectrum (e.g. van Daalen et al. 2011), there is yet to be investigated how these processes could affect the detectability of BAOs and the coupling between baryons and DM. We regard our work to be the first step in that direction.

We would like to highlight that the force resolution of our runs is a critical issue. A force resolution too high for a given mass resolution causes a spurious coupling between DM and baryons, and their respective clustering becomes severely affected, departing from the expected value 1 As we will show in the next subsection, this spurious coupling disappears only when the forces are softened on scales larger than the mean interparticle separation. For this reason, the main results of this paper will be based on simulations where forces are computed only with a PM method with a mesh of dimension equal to that of the unperturbed particle mesh, i.e. using a grid of $1024^{3}$ points to solve the Poisson equation. We present tests in this regard in the next subsection.

\subsection{Force softening and discreteness effects}

We now present the results of a suite of test runs, which serves as an illustration of the spurious coupling between baryons and DM discussed above. Fig. 1 shows the relative difference in the clustering of baryons and DM at $z=19$, as predicted by our $L=500 h^{-1} \mathrm{Mpc}$ simulations. We plot results from

\footnotetext{
1 Interestingly, we note that the total mass power spectrum always displays the correct amplitude and evolution, independently whether separately the DM or baryons have the correct power spectrum or not. Both components seem to collude to preserve the total mass power spectrum.
}

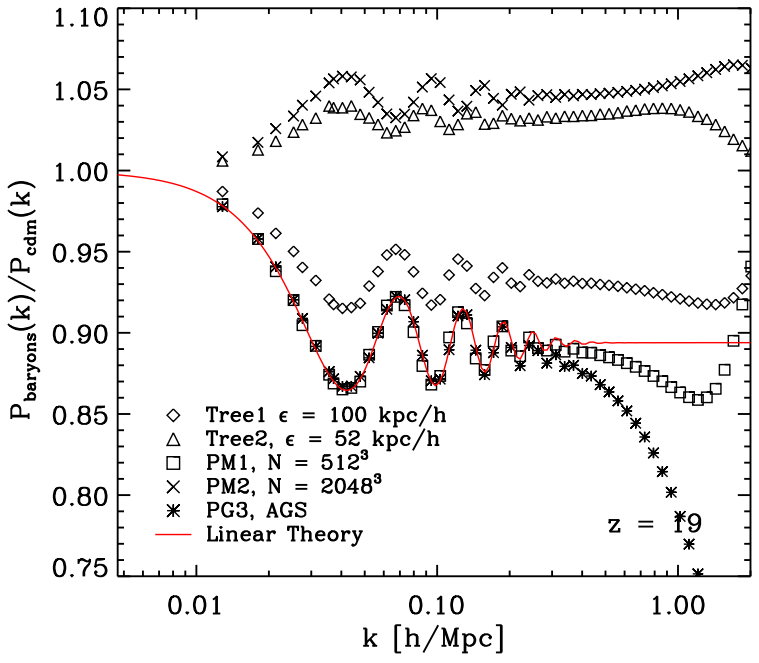

Figure 1. A test of the effect of force resolution in N-body simulations. The plot compares the ratio of the power spectrum of baryons and CDM at $z=19$ for four runs with different force resolution. Triangle and diamonds show results for TreePm runs with two different softening lengths, $\epsilon$, corresponding to $100 h^{-1} \mathrm{kpc}$ and $52 h^{-1} \mathrm{kpc}$, respectively. Squares and crosses show runs where forces are computed using only a particle-mesh (PM) method with cell sizes equal to $\sim 4 h^{-1} \mathrm{Mpc}$ and $\sim 1 h^{-1} \mathrm{Mpc}$ (corresponding to 1 and 0.25 times the mean inter-particle separation). Asterisks correspond to a Tree-PM run where the force acting on every particle is softened adaptively using a SPH kernel whose size is set by the distance to the $32 \mathrm{nd}$ nearest neighbour. Finally, the red solid line displays the expectation of linear perturbation theory.

different runs featuring different force resolutions and methods to compute the gravitational forces. The expectation is set by linear perturbation theory and it is displayed by a solid red line.

Test runs denoted as "Tree1" and "Tree2" (diamonds and triangles, respectively) show results for a standard TreePM force calculation and with Plummer-equivalent softening length, $\epsilon$, set to $1 / 20$-th and $1 / 10$-th of the mean inter-particle separation. Despite these being values adopted by state-of-theart simulations, in our case they overestimate the strength of the coupling between our two particle species providing results completely inconsistent with linear theory.

The same occurs in another test case, denoted as "PM2" (crosses), where we computed forces using only the PM method where the cell size is equal to $1 / 4$-th of the mean inter-particle separation of each particle type. As in the previous test cases, the ratio of the power spectra appears to be significantly higher than the prediction of linear theory. Although not displayed in this figure, we note that these simulations depart even further from linear theory at lower redshifts. A similar behaviour of incorrect large-scale growth has been reported by O'Leary \& McQuinn (2012).

We also show the results of another run, denoted as "AGS" (asterisks), where forces between baryon and DM particles (not however the intra-species forces) are softened adaptively using an SPH kernel with a width set by the distance to the 32nd neighbour (see Apendix A of Springel et al. 2001, for a discussion of this standard feature of Gadget). Unlike the previous runs, this one seems to correctly recover the relative large-scale clustering of baryons and DM. However, this comes 
at the expense of a strong suppression of the growth of small scales, resulting from a large smoothing of the force field (at high redshifts, our SPH kernel size corresponds to $32^{1 / 3} \sim 3$ times the mean-interparticle separation). We note that the use of an adaptive softening length (e.g. Iannuzzi \& Dolag 2011) should behave in a similar manner. Also, adaptive softening is inherent in all cosmological adaptive mesh refinement codes such as Ramses (Teyssier 2002) and ENZO (Bryan \& Norman 1997; O'Shea et al. 2004), so that a spurious coupling between the two species is not expected to occur. For these codes, the numerical evolution of the baryons on a grid comes however at the expense of numerical diffusion at the grid scale, leading to a comparable suppression of high- $k$ modes in the baryons (see Figure 23 of Hahn \& Abel 2011) as those in the adaptive $\mathrm{SPH}$ run.

Finally, we can see that the "PM1" run (squares) also reproduced correctly the linear growth. In this particular set-up the force has been smoothed on scales below the mean interparticle separation for each component separately (or 1.25 times the inter-particle separation for both components together). Here, the agreement with linear theory extends up to $k \sim 0.2 h \mathrm{Mpc}^{-1}$. On smaller scales, we see a downturn caused by the smoothing in the force field introduced by the Fourier mesh. Nevertheless, this smoothing is not as strong as that seen in the "AGS" case, and reaches the correct behaviour up to smaller scales. For this reason, we will adopt this particular numerical configuration for the simulations used in the remainder of the paper.

We can track the origin of the spurious coupling to the collissionality appearing in runs with high force resolution as a result of the discretisation of the density fields. On small scales, the force generated by N-body particles only approximately represents an homogeneous and continuous force field. This fact causes the particles to quickly couple on small scales, which affects the evolution even on large scales, increasing the rate at which differences in the clustering of baryons and DM dissipate.

We also note that we did not observe a significant impact of the choice of initial particle distribution on large-scale behaviour. While Yoshida et al. (2003) and Naoz et al. (2011), e.g., have argued for two randomly displaced glass distributions for the two species, we find that any choice of 'staggered' initial distribution - where separations between particles from the two species are locally maximized - reduces, but does not avoid, the discreteness effects. Regardless of the initial particle distributions, a very large force softening has to be chosen to obtain the correct growth. The reason why Yoshida et al. (2003) (and Hahn \& Abel 2011) do not observe a similar spurious coupling is most likely due to the significantly smaller boxes investigated there, where strong non-linear growth dominates quickly over the numerical artefacts.

It is important to note that the reason why discreteness effects in standard one- or two-component particle simulations are not as evident as in our case (e.g. Hamana et al. 2002) is because these simulations typically start from identical perturbation spectra, so that the spurious coupling cannot be easily diagnosed using the power spectrum. We argue, however, that in two-component simulations (such as $\mathrm{SPH}+\mathrm{DM}$ simulations), it should still appear as an additional binding energy between the two fluids that has to be overcome by pressure forces at late times. The impact is even less clear though, and harder to quantify, in standard one-fluid CDM calculations, but we expect it to appear as an artificial population of small-scale of low-mass halos, somewhat similar to those seen in warm DM simulations (e.g. Wang \& White 2007). Whether this has any sizeable and unforeseeable consequence for the results of simulations remains to be investigated.

\section{THE CLUSTERING OF BARYONS AND DARK MATTER}

Having identified a robust numerical setup, in this section, we present predictions for the large-scale clustering of baryons and dark matter as measured in N-body simulations. We start showing results in Fourier space (§3.1), then move to configuration space where we center our discussion on the BAO peak $(\S 3.2)$.

\subsection{Power Spectrum}

We begin by presenting in Fig. 2 the power spectra, $\mathrm{P}(\mathrm{k})$, of $\mathrm{DM}$ and baryons in real space, measured from our simulations at different output redshifts. Measurements were performed by mapping the particle distribution, using a clouds-in-cell scheme, onto a $1024^{3}$ grid and then Fast Fourier Transforming this field. We correct the effects of the assignment scheme by dividing each mode by the Fourier transform of a cubical tophat, but we do not subtract a Poisson shot-noise term. Here we show results from our large- and small-box simulations. The minimum wavenumber plotted is $2 \pi / 1000=0.0062 \mathrm{hMpc}^{-1}$ and $2 \pi / 250=0.025 \mathrm{hMpc}^{-1}$ for each of them.

The top panel of Fig. 2 compares the growth of total mass power spectrum from our runs with that predicted by linear perturbation theory, which is displayed as solid black lines. We note that we have evolved the $z=130$ linear theory power spectrum without considering the interaction of photons and baryons (effectively setting $\Omega_{r}=0$ ), which allows a more direct comparison with our simulations, where only gravitational interactions are considered.

We can see that our simulations closely reproduce the expected linear growth, differing only at the $4 \%$ level: The growth of the fundamental mode, from $z=130$ until $z=0$, is 10438.5, whereas the linear theory prediction is 10015.6. This close agreement supports the correctness of our numerical calculation. On small scales, the expected nonlinear growth dominates, and the measured $z=0$ power spectrum is a factor of $\sim 20$ larger than linear theory expectations at $k=1 \mathrm{hMpc}^{-1}$.

In the two bottom panels of Fig. 2 we plot the ratio between the power spectra of baryons and DM. This highlights the differences in the overall shape as well as in the BAO signature, visible in the range $0.1<\left(k / h \mathrm{Mpc}^{-1}\right)<0.3$. At all redshifts, the curves are systematically different from unity, which implies that the overall shape of the power spectrum of baryons and DM is different, even on very large scales. Density perturbations in the baryon density field are smaller than those in the DM at all times, resulting from the extra suppression produced by radiation pressure before recombination.

At the starting redshift of our simulations $(z=130)$, the amplitude of the power spectrum of baryons is $40 \%$ of that of the DM component, even on scales as large as the turn-over $\left(k=0.02 h \mathrm{Mpc}^{-1}\right)$. On smaller scales, the difference is constant down to the smallest scales that our simulations resolve. At later times, the gravitational interaction between baryons and DM particles couples the perturbation fields at all scales. As a result, both fields are homogenised and initial differences 

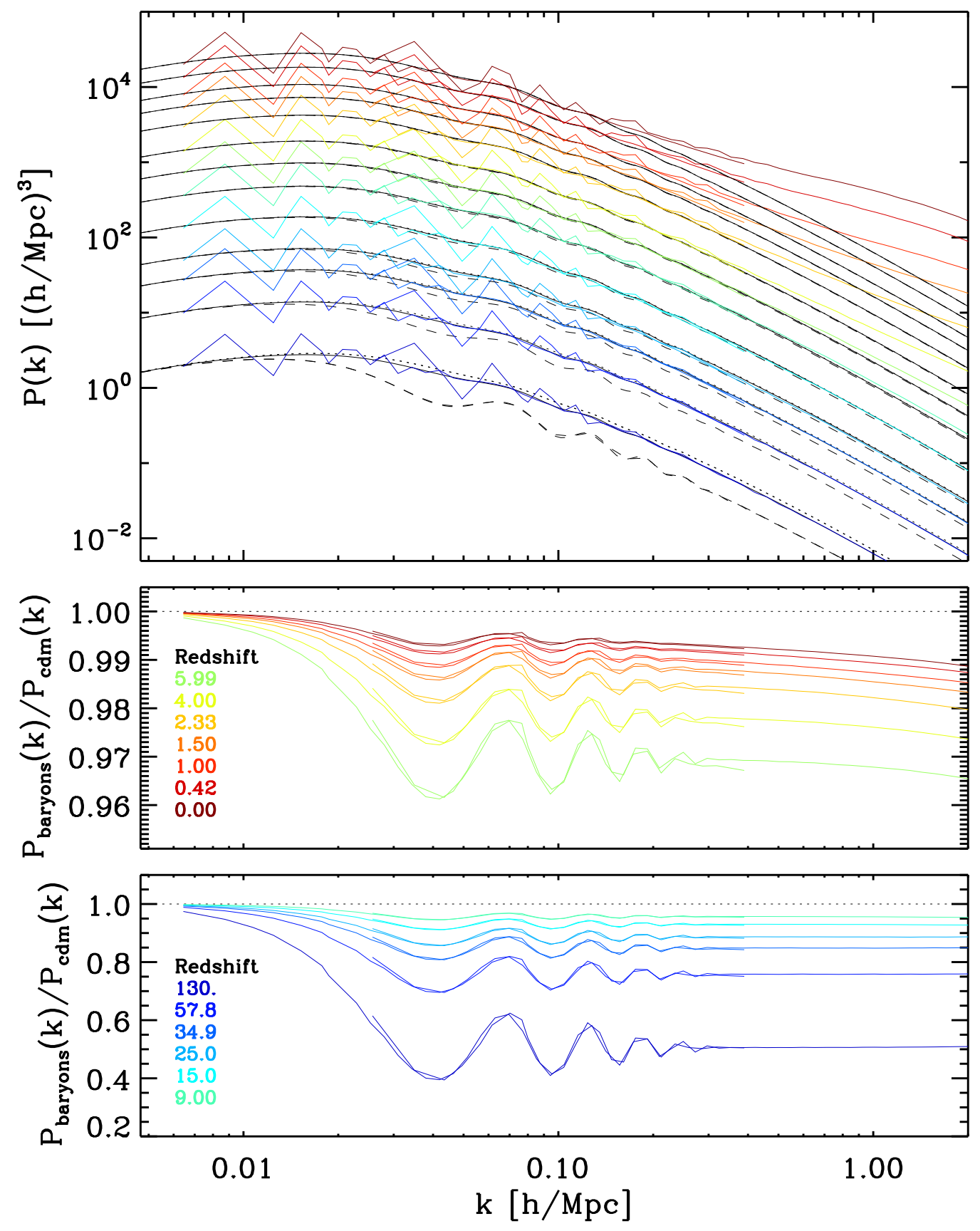

Figure 2. The growth of density fluctuations in the baryon and in dark matter fields, as measured by the power spectrum in real space. We show results for 13 redshifts, as indicated by the annotations in the figure, and for two different simulations containing the same number of particles but on boxes of side $1000 h^{-1} \mathrm{Mpc}$ or $250 h^{-1} \mathrm{Mpc}$. Their predictions overlap over the range $k=[0.03-0.3] h \mathrm{Mpc}^{-1}$. In the top panel, coloured lines show the measured total mass power spectrum in our simulations whereas dashed, dotted and solid lines show predictions of linear perturbation theory for the baryon, CDM and total mass power spectra, respectively. The lower panels show the ratio between the measured power spectra from baryons to that from CDM. Note that this ratio is not affected by the noise that arises from the finite sampling of large modes present in the simulation box. No Poisson shot-noise has been subtracted. Note the suppression of baryon perturbations relative to CDM perturbations at large $k$ that is due to non-linear effects.

are progressively reduced. At $z=35$ the baryon to $\mathrm{DM} \mathrm{P}(\mathrm{k})$ ratio is $\sim 0.85 \%$, and $\sim 0.95 \%$ at $z=9$. At the latter redshift, fluctuations have been amplified by a factor of 100 , but the differences between the baryon and DM power spectra are still approximately scale independent.
Gravitational interaction continues at lower redshifts, but, due to the effect of dark energy, perturbations grow more slowly than at higher redshifts. By $z=0$ the baryons power spectra show a $1 \%$ suppression compared to that of DM, almost the same difference present at $z=2$. The ratio now 




Figure 3. Comparison between simulation results, $R_{N-b o d y}$, and linear perturbation theory predictions, $R_{l t}$, for the relative difference between the power spectrum of baryons or CDM (top and bottom panel, respectively) to the total mass field: $R(k)=$ $P_{b, c d m} / P_{\text {total }}$. We show results from our $1 h^{-1} \mathrm{Gpc}$ box for $k<$ $0.3 \mathrm{hMpc}^{-1}$, and from our $250 \mathrm{~h}^{-1} \mathrm{Mpc}$ for smaller scales. Coloured lines show results at different redshifts, as indicated by the legend.

displays a scale dependence - small scales approach unity at a slower rate - which can be interpreted as the DM field having experienced more nonlinear evolution and mode coupling than the baryonic field. This could be a residual effect of the DM power spectrum having higher amplitude than that of baryons.

The amplitude of the oscillatory behaviour seen in the lower panels of Fig 2 arises from differences in the BAO feature. The bigger their amplitude, the more dissimilar the BAO are in the two fluids. At the starting redshift, oscillations are large, i.e. BAOs are very weak in the DM but very notorious in the baryons. (For baryons, the amplitude of BAO is $\sim 30 \%$ of that of the power spectrum, but for DM, it is only $\sim 5 \%$.) At lower redshifts, gravity will damp the BAO in the baryons and increase their amplitude in the DM. However, as in the case of the overall shape of the power spectra, even at $z=0$, there are still some residual differences. In the next subsection we will investigate the evolution of the shape and amplitude of BAO signal in more detail.

Fig. 3 shows a detailed comparison of our results with the prediction of linear perturbation theory. The top/bottom panel shows the ratio between the power spectrum of $\mathrm{DM} /$ baryons to that of the total mass, divided by the same ratio predicted in linear theory. Thus, departures from unity indicate where linear theory is not able to predict the relative differences, with respect to the total mass, present in the spatial distribution of DM or baryons. Firstly, we note that departures in this ratio are much smaller, and appear on smaller scales, than absolute deviations of each component with respect to linear theory. Here, differences are below $0.5 \%$ for the baryons and below $0.1 \%$ for the DM, to be compared with one order of magnitude deviations in their individual amplitude (c.f. Fig. 2). Hence, linear theory predicts this quantity at a remarkable accuracy. We note that our findings qualitatively agree with the analytical results of Somogvi \& Smith (2010), who, using renormalised perturbation theory extended to a multi-fluid case, also reported a positive deviation from unity on small scales for DM, and negative deviation for baryons.

Overall, from this figure we get a picture complementary to our earlier discussion: Nonlinear evolution produces a faster growth in DM perturbations relative to that in baryons. For this reason, the amplitude of the baryon spectrum is overestimated, whereas that of DM is underpredicted. This results in an overprediction of the baryon-to-DM power spectra ratio, which increases at smaller scales. Linear theory predicts only a $1 \%$ difference between the clustering of DM and baryons by $z=0$, whereas in our simulations we measure a $1.5 \%$ difference. These departures are small, but they should increase as we consider smaller scales and, nevertheless, exemplify that gravitational evolution can be followed at some degree by linear or higher-order perturbation theories, but once the density contrast reaches values well above unity, numerical simulations are needed to properly and accurately follow the growth of structure.

\subsection{The BAO peak in the Correlation Function}

We now complement the Fourier-space results by presenting the correlation function, $\xi(r)$, of baryons, DM and that of the total mass, as measured in our simulations. Exploring our results in configuration space allows us to focus on the BAO and also to have a more direct comparison with observational data and expectations from future galaxy surveys, which usually present their measurements in the form of correlation functions.

We compute correlation functions in Fourier space using a mesh of 1024 points per dimension, which provides an accurate estimation of correlation functions on $r \gtrsim 20 h^{-1} \mathrm{Mpc}$ for our $1 h^{-1} \mathrm{Gpc}$ box. This approach is considerably faster than a direct pair count when computing correlation functions on large scales in catalogues containing a large number of particles.

Fig. 4 shows the correlation functions computed in this way for both baryons and DM in our $1 h^{-1} \mathrm{Gpc}$ simulation. The top panel displays the measurements in real space while the bottom panel shows the results in redshift space using a planeparallel approximation (i.e. the effect of peculiar motions in distance estimators is included by considering an observer at infinity). We show results on scales $r=[60-135] h^{-1} \mathrm{Mpc}$ to focus on the BAO peak (appearing at $\sim 110 h^{-1} \mathrm{Mpc}$ ). Each measurement has been divided by the square of the growth factor, and additionally by the Kaiser "boost factor" (Kaiser 1987), in the case of redshift-space results. Therefore, if the growth of a given component is completely linear and scaleindependent, then curves at different redshifts would coincide in each panel. We see that this is indeed a good first-order approximation, though systematic differences exist. We discuss this next.

In the top-left panel of Fig. 4 we can see how the BAO peak is gradually imprinted in the DM distribution. At $z=$ 130 , the correlation function is still close to a power-law with just a relatively small bump at $110 h^{-1} \mathrm{Mpc}$. However, in an extremely rapid process, the $\mathrm{BAO}$ emerges and reaches almost its full amplitude by $z \simeq 30$, only $\sim 100$ Myrs after. During this period, we also see a small scale-dependent suppression of the correlation function at scales $r<80 h^{-1} \mathrm{Mpc}$, to accommodate for the growing peak. The subsequent evolution happens 



Figure 4. Real-space (top) and redshift-space (bottom) correlation functions in our simulations at different redshifts, focusing in the BAO peak. Measurements are shown for baryons, dark matter and total mass (panels (a), (b) and (c)), and for linear bins of $\Delta r=3 h^{-1} \mathrm{Mpc}$. Results were scaled by the square of the appropriate growth factor, $D(z)$, and, in the case of the redshift-space correlation functions, additionally by the Kaiser boost factor: $f \equiv 1+\frac{2}{3} \beta+\frac{1}{5} \beta^{2}$, where $\beta \approx \Omega(z)^{0.55}$.

at a lower rate, and the maximum relative amplitude of the $\mathrm{BAO}$ is reached at $z \sim 6$, the moment in which mild nonlinear coupling of independent Fourier modes start to have a noticeable effect, decreasing again the amplitude of BAO peak and broadening its shape (e.g. Angulo et al. 2005).

The history of baryons, displayed in the middle panels, is the opposite. At the starting redshift the correlation function is dominated by the BAO peak, it is $\sim 4$ times larger than the broad-band shape of the correlation function, and a factor of $\sim 2$ larger than the maximum relative amplitude ever present in the total mass field. This, however, quickly changes, with the amplitude of the peak decreasing linearly with redshift. As in for the DM case, density fluctuations in the baryons also show a scale-dependent growth, but in the opposite direction.

The evolution in baryons and DM exactly compensate each other to give an almost perfectly scale-independent growth of fluctuations in the total mass density, as can be seen in the right panels of Fig. 4 This behaviour is only broken at low redshift by non-linear mode coupling, which smears out the BAO peak (e.g. Angulo et al. 2008; Sánchez et al. 2008, and references therein). The evolution is compensated since perturbations do not grow independently, but are linked through a common gravitational potential that determines an identical acceleration field for both components. Thus, gravity acts as a homogenizer of the fields. In fact, although by $z=0$ there are residual differences, if we let the simulation run into the future, then eventually both fields will be indistinguishable from each other on large scales.

The bottom panel of Fig. 4 shows the clustering in redshift space (a quantity closer to that observed by spectroscopic galaxy surveys) for baryons, DM and for the total mass. For both components, and at all redshifts, we can see that the measured correlation functions are not simply a scaled version of their real-space counterpart, as one might naively expect in linear theory.

At high redshift, the redshift-space enhancement in the overall correlation function is smaller than linear theory expectations for DM, but larger for baryons. At low redshifts, predictions are closer to our measurements, and the respective curves therefore overlap better in this plot. At the same time, the BAO peaks get damped as a consequence of nonlinear contributions to peculiar velocities. At high redshifts, this is also true for baryons but not for the DM field, which sees an increase in the amplitude of the BAO peak.

All the differences described above are summarised and quantified in Fig. 5 which displays the correlation function for baryons and DM relative to that of the mass for different redshifts and for both real and redshift space, averaged over two scales. In the top panel, averaged between 65 and $75 h^{-1} \mathrm{Mpc}$, capturing changes in the overall amplitude. In 



Figure 5. The redshift evolution of the average correlation function for baryons and DM relative to that of the total mass. In the top panel, we show the ratio of the correlation functions averaged between 65 and $75 h^{-1} \mathrm{Mpc}$ separations. Thus, this displays differences in the overall amplitude of the respective correlation function. In the bottom panel, we perform the average between 105 and $110 h^{-1} \mathrm{Mpc}$. Thus this can be regarded as the evolution in the amplitude of the BAO peak for baryons and DM.

the bottom panel, between 105 and $110 h^{-1} \mathrm{Mpc}$, so it shows changes in the amplitude of the BAO peak.

We see that the correlation function amplitude at $z=10$, relative to that of the total mass, is $\sim 1 \%$ higher for the case of $\mathrm{DM}$, and $\sim 3 \%$ smaller for baryons. These figures are reduced to a sub-percent level by $z=0$. The differences are largest for the BAO peak in the baryons: $\sim 10 \%$ excess at $z=10$, which is reduced to about 1 percent by $z=0$. This effect is smaller than those introduced by galaxy formation physics (Angulo et al. 2013, in prep.), which could cause up to $\sim 10 \%$ effects.

Redshift space distortions (RSD) reduce the amplitude of the BAO peak in the baryons at all redshfts, relative to that in real space. For DM, they enhance the BAO peak at high redshifts, but damp it after $z \sim 1$. The observed behaviour is explained by the fact that the gravitational potential that originates the coherent velocity flows is generated by the total mass distribution, which is not identical to either the baryonic or DM material but to their mass weighted average. Thus, the density-velocity relation expected in linear theory does not hold separately for either of the components. At high redshift this homogenises the field further at the BAO scale, whereas at low redshift nonlinear RSD dominate and diffuse the peak.

\subsection{Comparison with 1-fluid simulation}

Finally, we test the widespread assumption that the nonlinear evolution of perturbations in the density field of baryons and DM can be approximated as a single fluid representing the total mass field. For this, we run our two N-body simulations with exactly the same numerical configuration, but this time baryons and DM have identical primordial density and velocity
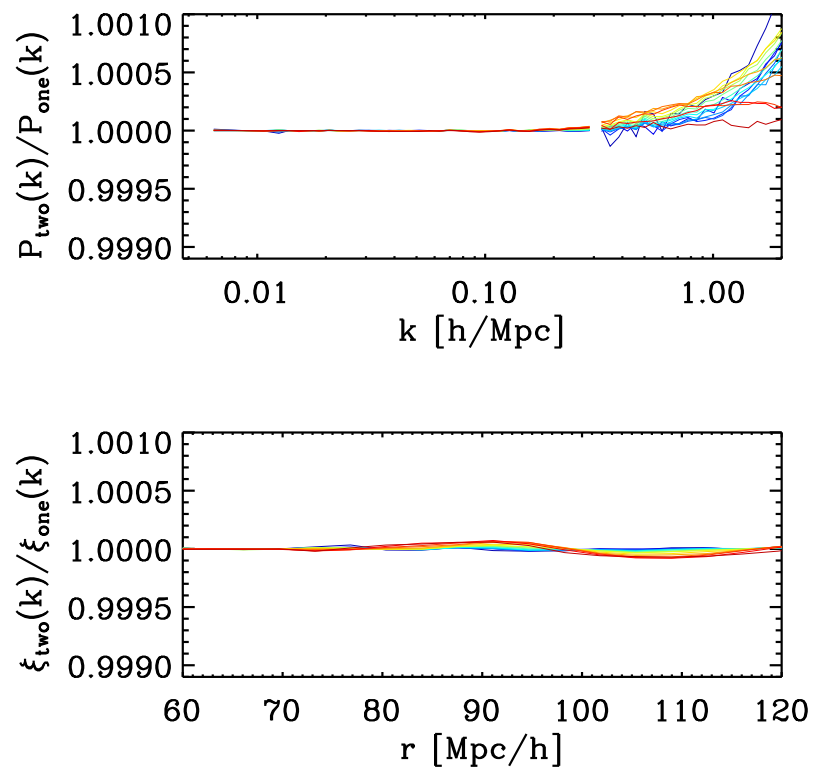

Figure 6. Comparison of the power spectrum (top panel) and correlation function (bottom panel) predicted by our two-fluid simulations with those predicted by a single-fluid simulation, in which the initial conditions of particles were drawn following the total mass power spectrum at the starting redshift of our simulations. Lines of different colours display results at different redshifts, following the same convention as in Fig. 3

power spectra (equal to that of the total mass), so that they effectively behave as a single fluid. With this we can test to what degree the nonlinear evolution of two interacting fluids is equivalent to that of a single fluid with the mass-weighted average power spectrum.

Fig. 6 presents our results. In the top panel we show the ratio of the power spectra of our two- and one-fluid simulations. On large scales, both simulations are virtually indistinguishable, it is only in the nonlinear regime where differences appear. The one fluid case underestimates the amount of nonlinear clustering in a roughly redshift-independent manner. The differences are small: at $k \lesssim 2 h \mathrm{Mpc}^{-1}$ are less than $0.1 \%$, although they increase exponentially with the wavenumber $k$ to reach percent level by $k \sim 10$. Unfortunately, our simulations lack the spatial resolution to pin down this more accurately. Our results agree with the analytical findings of Somogyi \& Smith (2010), who found a discrepancy of less than $\sim 0.3 \%$ at $k=1 \mathrm{hMpc}^{-1}$ between the mass power spectra from one- and two-fluid calculations at $z=0$.

In the bottom panel of Fig. 6 we display an analogous comparison but focusing on the BAO peak. Contrary to the Fourier-space behaviour, we see that the amount of nonlinear diffusion of the BAO peak is larger in the one fluid case. Nevertheless, the differences are extremely small: below $0.01 \%$. These results validate the use of one-fluid simulations to study the large-scale distribution of mass, and hence of the BAO signal, in the Universe. Although not shown in the figure, we have also checked that results in redshift space present equally small differences.

In this section we focused on two-point statistics, but higher-order statistics, in principle, are also be affected. However, we do not expect to find differences considerably higher than those shown for the power spectrum and correlation func- 
tion. This, given the very small discrepancies we see between 1 and 2-fluid simulations in the nonlinear regime, and that these nonlinear interactions are the responsible for higher order correlations of the density field.

\section{SUMMARY AND CONCLUSIONS}

We have performed a direct calculation of the gravitational coupling of baryons and dark matter, from $z=130$ to $z=0$, using N-body simulations. We focused primarily on large scales to explore how the BAO signature, initially present primarily only in the spatial distribution of baryons, is imprinted in the DM distribution.

At the starting redshift, the density and velocity power spectra of baryons and DM differ considerably. At latter times, gravity acts as a diffusive agent of the primordial clustering differences. We find that the bulk of the differences are dissipated quickly, but also that this process is not fully completed by the present day. Differences in the power spectrum amplitude of $\sim 40 \%$ at $z=130$ are brought down to sub-percent level by $z=0$. Interestingly, the BAO peak does not evolve as quickly and differences are still above $1 \%$ at low redshifts.

On large scales, our results are consistent with linear perturbation theory: the ratio between the power spectra of baryons and DM decreases in a roughly scale-independent manner at high redshifts. On small scales, $k \gtrsim 0.2 \mathrm{hMpc}^{-1}$, nonlinear evolution breaks this and we predict a growth of the ratio between the baryon and the DM power spectrum that is slower than in linear theory. However, the size of these departures is small (below 0.5\%), thus, in practice, linear theory is a very accurate predictor of all these effects.

Additionally, we confirmed that the nonlinear evolution of the total mass power spectrum can be accurately predicted by numerical simulations treating all mass as a single fluid with a single power spectrum. Most of these results are in qualitative agreement with the analytical work of Somogyi \& Smith (2010).

We conclude that assuming that baryons and dark matter have the same spatial distribution on the BAO scale is a good approximation given the accuracy with which it is expected to be measured by upcoming large galaxy surveys. However, we emphasize that the quality of this assumption is redshiftdependent, being worse at high redshifts and better at low redshifts. The amplitude in the BAO peaks differs at the $10 \%$ level at $z=10$, but only at $1 \%$ at $z=0$. The differences at low redshifts are smaller than the effects expected to be introduced by galaxy formation physics, but they could perhaps eventually be detected in the future in, for instance, HI clustering from SKA measurements, or in another. If this is the case, our work indicates that at high redshift the bias of tracers that are sensitive to baryons instead of the total matter distribution might include also a non-negligible baryon bias, though more work in this respect is needed, since the size of the effect likely depends on the type of tracer and object selection criteria employed.

We note that it is certainly possible that the distortions discussed in our paper could result into a systematic bias in cosmological parameters extracted from BAO measurements. These are, however, hard to predict in practice due to several reasons. The first one is a theoretical issue, and has to do with the question of whether galaxies will follow the baryons, the DM, or the total mass. In other words, is the galaxy bias simplest when expressed with respect to the underlying DM, baryon or mass clustering? The second issue has to do with how the cosmological parameters are actually measured. For instance, at low redshifts, distortions in the amplitude are very degenerated with the amount of nonlinear evolution in the BAO peak. Thus, amplitude changes will likely not introduce any biases in the measured sound horizon scale. At higher redshifts this is not true, and the effects discussed here should be considered In any case, for a quantitative assessment, all the details of a particular observational campaign should be taken into account. We anticipate that this will be an interesting area of research in the future.

Finally, we highlighted that force resolution is a critical issue to obtain accurate results for simulations with two fluids with distinct primordial density fluctuations. Discreteness effects in standard numerical configurations resulted in an artificial coupling between baryons and DM particles, which propagated to large scales and lead to an artificially fast growth of large-scale modes. We tracked this issue to the loss of collisionallity in the fluids, which, thus, could be solved only by softening gravitational forces below the mean inter-particle separation. The numerical artefacts arising from a high force resolution were evident in our runs, but they should also be present in standard N-body calculations. However, while the net effect is not clear, it clearly warns for a careful assessment of the robustness of current numerical simulations in the mildly non-linear regime.

\section{ACKNOWLEDGEMENTS}

REA acknowledges useful discussions with T. Naab, A. Sanchez, V. Springel and S. White. We would also like to thank Naoki Yoshida, Robert Smith and Simon White for valuable suggestions and comments on the draft. OH acknowledges support from the Swiss National Science Foundation (SNSF) through the Ambizione fellowship. TA acknowledges support by the LDRD program at the SLAC National Accelerator Laboratory as well as the Terman fellowship at Stanford University. We gratefully acknowledge the support of Stuart Marshall and the SLAC computational team, as well as the computational resources at SLAC.

\section{REFERENCES}

Angulo R., Baugh C. M., Frenk C. S., Bower R. G., Jenkins A., Morris S. L., 2005, MNRAS, 362, L25

Angulo R. E., Baugh C. M., Frenk C. S., Lacey C. G., 2008, MNRAS, 383, 755

Angulo R. E., Springel V., White S. D. M., Jenkins A., Baugh C. M., Frenk C. S., 2012, MNRAS, 426, 2046

Bernardeau F., van de Rijt N., Vernizzi F., 2012, Phys. Rev. D, 85, 063509

Beutler F. et al., 2011, MNRAS, 416, 3017

Blake C. et al., 2011, MNRAS, 418, 1707

Bryan G. L., Norman M. L., 1997, in Astronomical Society of the Pacific Conference Series, Vol. 123, Computational Astrophysics; 12th Kingston Meeting on Theoretical Astrophysics, Clarke D. A., West M. J., eds., pp. 363-+

Chang T., Pen U., Peterson J. B., McDonald P., 2008, Physical Review Letters, 100, 091303

Cole S. et al., 2005, MNRAS, 362, 505 
Cooray A., 2002, in Astronomical Society of the Pacific Conference Series, Vol. 283, A New Era in Cosmology, Metcalfe N., Shanks T., eds., p. 162

Eisenstein D. J. et al., 2005, ApJ, 633, 560

Hahn O., Abel T., 2011, MNRAS, 415, 2101

Hamana T., Yoshida N., Suto Y., 2002, ApJ, 568, 455

Hu W., Dodelson S., 2002, ARA\&A, 40, 171

Iannuzzi F., Dolag K., 2011, MNRAS, 417, 2846

Kaiser N., 1987, MNRAS, 227, 1

Kitaura F.-S., Gallerani S., Ferrara A., 2012, MNRAS, 420, 61

Komatsu E. et al., 2011, ApJS, 192, 18

Kuhlen M., Vogelsberger M., Angulo R., 2012, Physics of the Dark Universe, 1, 50

Ma C.-P., Bertschinger E., 1995, ApJ, 455, 7

Mao X., Wu X., 2008, ApJ, 673, L107

Naoz S., Yoshida N., Barkana R., 2011, MNRAS, 416, 232

O'Leary R. M., McQuinn M., 2012, ApJ, 760, 4

O'Shea B. W., Bryan G., Bordner J., Norman M. L., Abel T., Harkness R., Kritsuk A., 2004, arXiv:astro-ph/0403044 Rhook K. J., Geil P. M., Wyithe J. S. B., 2009, MNRAS, 392, 1388

Sánchez A. G., Baugh C. M., Angulo R. E., 2008, MNRAS, 390,1470

Scoccimarro R., 1998, MNRAS, 299, 1097

Somogyi G., Smith R. E., 2010, Phys. Rev. D, 81, 023524

Springel V. et al., 2005, Nature, 435, 629

Springel V., Yoshida N., White S. D. M., 2001, New Astronomy, 6, 79

Teyssier R., 2002, A\&A, 385, 337

van Daalen M. P., Schaye J., Booth C. M., Dalla Vecchia C., 2011, MNRAS, 415, 3649

Wang J., White S. D. M., 2007, MNRAS, 380, 93

White M., Pope A., Carlson J., Heitmann K., Habib S., Fasel P., Daniel D., Lukic Z., 2010, ApJ, 713, 383

Yoshida N., Sugiyama N., Hernquist L., 2003, MNRAS, 344, 481

Zel'Dovich Y. B., 1970, A\&A, 5, 84

Zhan H., Wang L., Pinto P., Tyson J. A., 2008, ApJ, 675, L1 